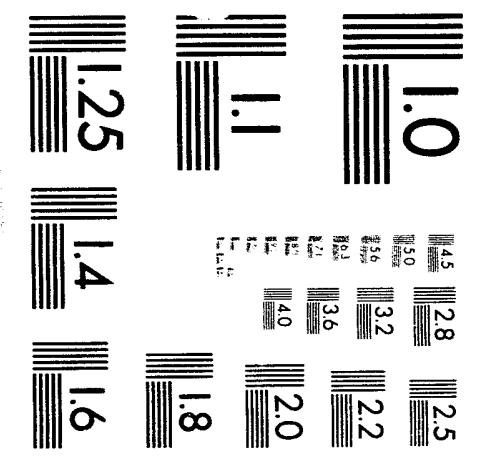



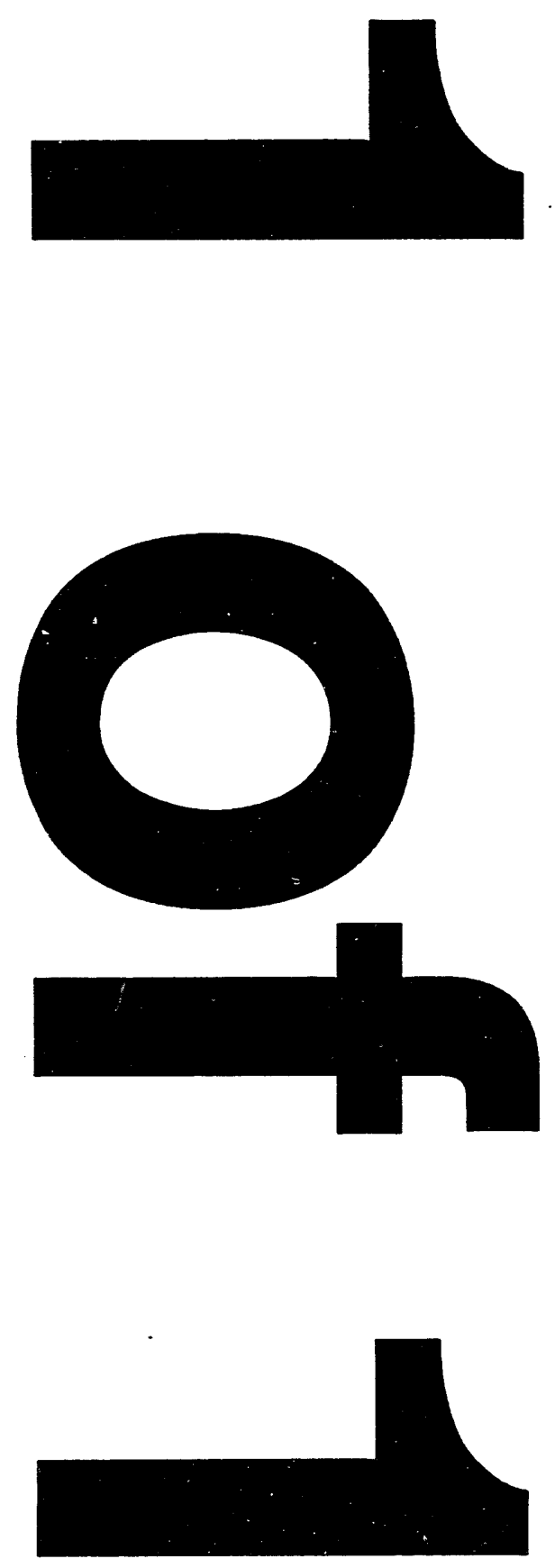


\title{
IMPLEMENTATION OF A QUASI-REALTIME DISPLAY OF DIII-D NEUTRAL BEAM HEATING WAVEFORMS
}

\author{
by \\ J.C. PHILLIPS
}

This is a preprint of a paper to be presented at the 15th IEEE Symposium on Fusion Engineering, October 11-15, 1993, Cape Cod, Massachusetts, and to be printed in the Proceedings.

\author{
Work supported by \\ U.S. Department of Energy \\ Contract DE-AC03-89ER51114
}

GENERAL ATOMICS PROJECT 3466 OCTOBER 1993

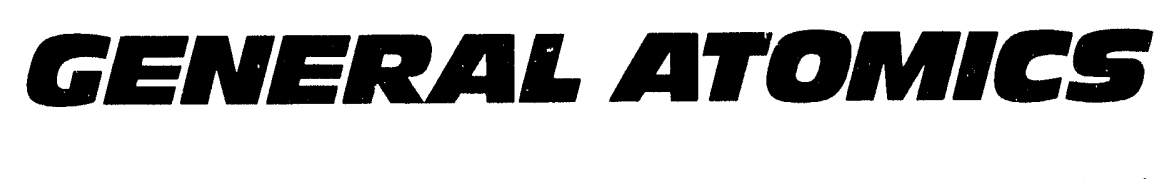




\title{
IMPLEMENTATION OF A QUASI-REALTIME DISPLAY OF DIII-D NEUTRAL BEAM HEATING WAVEFORMS
}

\author{
J.C. Phillips \\ General Atomics \\ P.O. Box 85608, San Diego, California 92186-9784
}

\begin{abstract}
The DIII-D neutral beam system employs eight $80 \mathrm{keV}$ ion sources mounted on four beamlines to provide plasma heating to the DIII-D tokamak. The neutral beam system is capable of injecting over $20 \mathrm{MW}$ of deuterium power with flexibility in terms of timing and modulation of the individual neutral beams. To maintain DIII-D's efficient tokamak shot cycle and make informed control decisions, it is important to be able to determine which beams fired, and exactly when, by the time the tokamak shot is over. Previously this information was available in centralized form only after a several minute wait. A costeffective alternative to the traditional eight-channel storage oscilloscope has been implemented using off the shelf PC hardware and software. The system provides a real time display of injected neutral beam accelerator voltages and tokamak plasme current, as well as a summation waveform indicative of the total injected power as a function of time. The hardware consists of a Macintosh Centris $650 \mathrm{PC}$ with a Motorola 68040 microprocessor running at $20 \mathrm{MHz}$. Data acquisition is accomplished using a National Instrument's 16-channel analog to digital conversion board for the Macintosh. The color displays and functionality were developed using National Instruments' LabView environment. Because the price of PCs has been decreasing rapidly and their capabilities increasing, this system is far less expensive than an eight-channel strrage oscilloscope. As a fexible combination of $P C$ and software, the system also provides much more capability than a dedicated oscilloscope, acting as the neutral beam coordinator's logbook, recording comments and availability statistics. Data such as shot number and neutral beam parameters are obtained over the local network from other computers and added to the display. Waveforms are easily archived to disk for future recall. Details of the implementation will be discussed along with samples of the displays and a description of the system's function and capabilities.
\end{abstract}

\section{INTRODUCTION}

DIII-D, a tokamak research device operated by General Atomics for the U.S. Department of Energy, is one of the mc st flexible and best diagnosed tokamaks in the world. Discharges can be formed on a ten-minute cycle, and a typical run day will have 40 to 60 discharges. Separate computers are used for control of the tokamaix and acquisition of the experimental data. Operating in this pulsed mode, once the discharge is complete, data for hundreds of power supply, magnetics, and diagnostic waveforms must be collected from local digitizer memories and processed through one computer. Recent DIII-D discharges have resulted in shot files of over $90 \mathrm{MB}$ [1]. This has meant that viewing of data handled by these machines has become less and less of a "real-time" experience, and, in fact, waits of several to many minutes are more common. These waits are necessary because typically all data is acquired from an assortment of digitizers and diagnostic computers and then processed into a large shot file located on a remote VAX cluster, where it becomes available for viewing. Further delays are induced if the data of interest requires additional calculations and scaling to be performed on it.

To provide heating of the tokamak discharges, DIII-D employs over $20 \mathrm{MW}$ of neutral beam power supplied by eight $80 \mathrm{keV}$ ion sources. The sequencing of this power can be changed on a discharge to discharge basis, and it is important to view the performance for the current discharge in order to make informed decisions for the next.

In the case of the neutral beam data, handled by four independent MODCOMP Classic computers, two to three minutes elapse before the waveforms may be viewed. Much of this wait is because over $4 \mathrm{MB}$ of data are collecied from digitizer-local memory over serial CAMAC highways for each MODCOMP. Even then, the waveforms are viewable only on four separate screens with no common time reference. Each neutral beam may be skewed in time with respect to the other, but the data on each MODCOMP are displayed only with respect to the "time zero" of that beam. A great improvement was made when an IBM PC system was developed [2] to display selected beam parameters, including a representation of requested and actual beam performance. This display is well used and was the only central point of reference in the beam area. Unfortunately, this display too must wait for the MODCOMP data acquisition cycle to complete and thus cannot generate its display until two to three minutes after the shot.

To satisfy the demand for the ability to determine whether the neutral beams performed to specification as soon as possible after the DIII-D shot is complete, a digitization system has been developed for the microcomputer used during operations by the neutral beam coordinator. The coordinator has traditionally used this

Mamiscript received October 12, 1893. This is a report of work aponsored by the U.S. Department of Energy under Contract No. DE-AC03-89ER51114. 
computer for logging of shot by shot availability statis tics and report generation, but is now also able to view the combined neutral beam waveforms. While a few years ago this would probably have been achieved with a storage oscilloscope, the approach adopted here has been both more cost effective and more flexible, both in terms of function and appearance. The "channels" for this computer based display are easily and clearly labeled, and the display is in color for ready identification of traces at a glance.

\section{FUNCTION}

Functionally, the system acquires and displays nine waveforms, consisting of eight neutral beam accelerator voltages and DIII-D plasma current, while also performing other tasks such as word processing and running a spreadsheet. The system is flexible in that more than nine data channels could be acquired, the appearance and size of the 'scope display' can be changed or resized, all with very little programming effort. In comparison to the alternative of a traditional storage oscilloscope, the system offers far more capability as it can be used by the neutral beam coordinator for logging their daily reports and tracking availability statistics. The data display portion requires no operator attention. The data acquisition software waits for a trigger indicating that a DIII-D shot is in process, automatically acquires the data and updates its display. In addition, the display shows the DIII-D shot number and time of day for the current shot. It is a peculiarity of the DIII-D control room that the neutral beam coordinator cannot directly see the DIII-D shot number display, or the oscilloscope which displays beam and plasma waveforms. This new display has eased both problems.

\section{HARDWARE}

The neutral beam waveform data, along with DIII-D plasma current, are acquired using a National Instruments NB-MIO-16-H Data Acquisition board, which uses a 12 bit ( 1 in 4096) analog to digital multiplexed converter. While the board is capable of digitizing at a $100 \mathrm{kHz}$ rate, for this relatively "slow" application, a sampling rate of $1 \mathrm{kHz}$ was chosen. Data is acquired for a ten-second sweep, which can easily be lengthened should DIII-D extend its operating pulse length. The data acquisition board is configured for 16 single-ended inputs (as opposed to eight differential), allowing more than enough channels for the eight neutral beams and DIII-D plasma current. The board is resident in a Macintosh Centris 650 PC. The Centris 650 was chosen because it provides an economical balance, on the one hand offering three expansion slots (one of which is used by the data acquisition board), a fast $20 \mathrm{MHz} 68040$ 32-bit microprocessor with integral Floating Point Unit, built-in support for a 17 or 19 in. monitor, and, on the other hand, costing significantly less than the top of the line "Quadra" series Macintoshes. The hardware configuration is shown in Fig. 1. The data is displayed on a $17 \mathrm{in.} \mathrm{non-interlaced}$ high resolution video monitor.

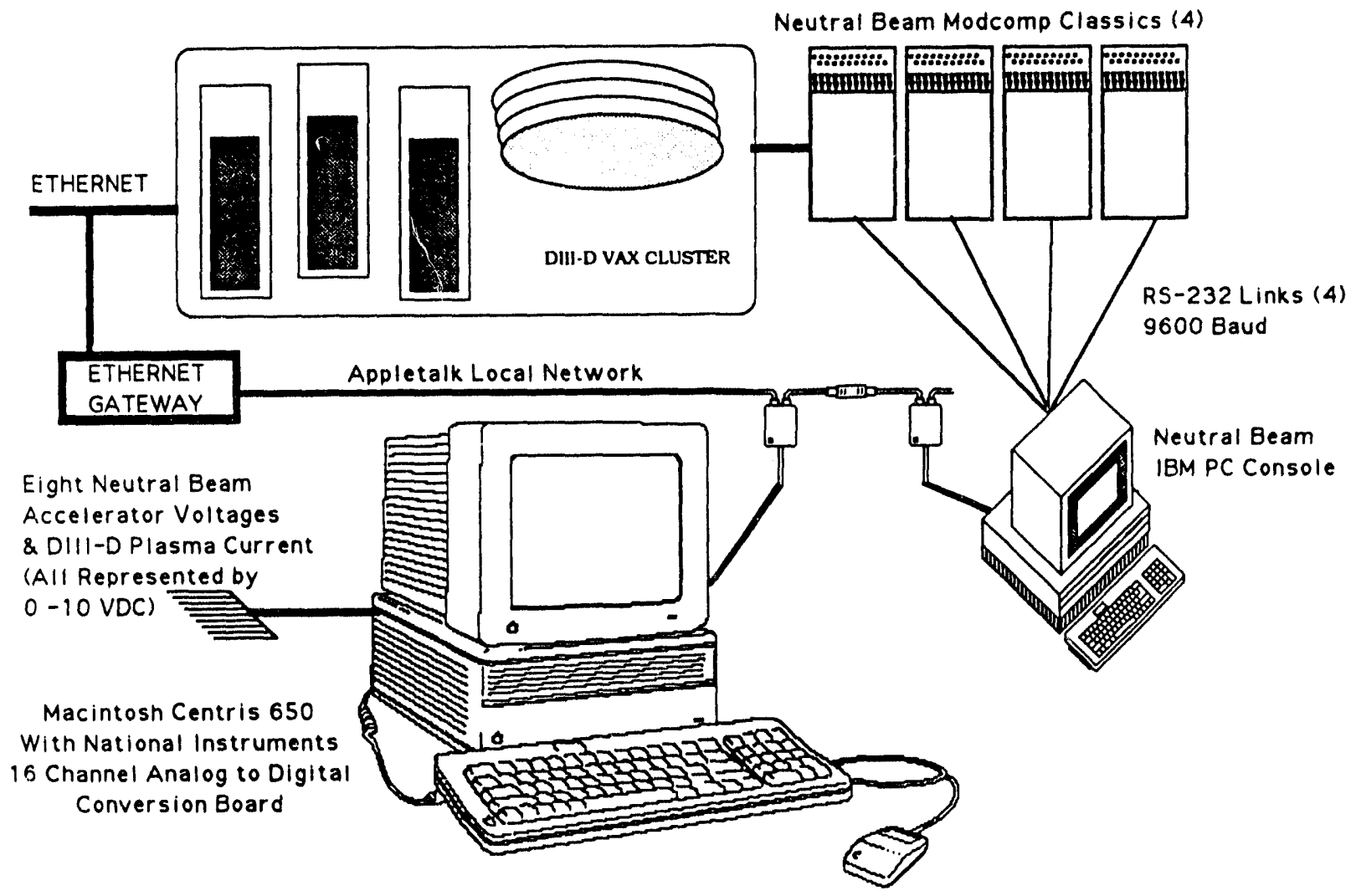

FIg 1. The data acquisition hardware. 


\section{PROGRAMMING}

Given that resources are always limited, it is important that effort expended programming the data acquisition and associated display will be applicable to other work. Programming was done using National Instruments' LabView software. The very stark difference between this environment and more traditional approaches is illustrated in Fig. 2. Fig. 2(a) shows a code segment from a procedural language such as ' $\mathrm{C}$ ' and merely sets up a "for loop" assigning each element of an array to the iteration of the loop. Fig. 2(b) shows the equivalent operation in LabView. To program this example the icons are merely selected from a palette and "wired" into place. A slightly more complex example, that of setting and waiting for a trigger is shown in Fig. 3. While having some ' $C$ ' experience, the author had no previous practical experience with LabView but was still able to get the basics of this project working within three days. Two versions of LabView are sold, one a development system and the other a less expensive run-time only version. In the development version, compilation is essentially transparent to the user, occurring in a few seconds and only if there have been changes to the "code." Once developed and complete, the application can be run by the less expensive run-time only version. Functional blocks of code written with LabView are called Virtual Instruments (VIs). Once a VI is written, it can be ported into other VIs, even into other projects. If there is a function for which no icon or VI exists, then the programmer is still free to write code in a language such as ' $C$ ' and incorporate it in a project as a VI.

While originally an environment available only for Macintosh, LabView now runs with virtually identical appearance on IBM 386 and 486 AT computers running Microsoft Windows, as well as on SUN SPARCstations [3]. Data acquisition for all of these platforms can be by means of GPIB bus, VXI crates, serial port, CA$\mathrm{MAC} / \mathrm{GPIB}$, as well as using dedicated boards (as in

$$
\begin{aligned}
& \text { main() } \\
& \text { int } i, x[16] ; \\
& \text { for }(i=0 ; i<=15 ; i++) \\
& \quad x[i]=i ;
\end{aligned}
$$

Fig. 2. (a) A simple "for" loop in a procedural language such as 'C,' assigning the value of each iteration of the loop to an array of integers. (b) The same operation shown in LabView. Standard icons are selected from palettes and wired together.

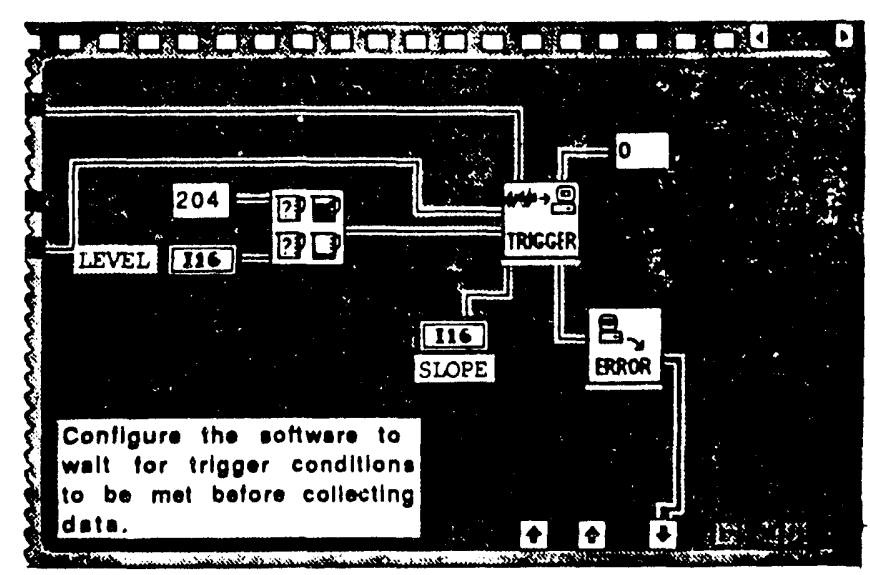

Fig. 3. The bulk of the work of this segment of LabView code is done by pre-existent modules, indicated by the "level comparator" and "trigger" modules, as well as an "error message decoder" which looks up a numerical error code and generates a representative text string.

this project). This means that the investment in learning how to program in this environment now has broad application across several different platforms and hardware configurations.

\section{NETWORKING}

This microcomputer is attached to the AppleTalk local area network and will soon be connected directly to the DIII-D Ethernet network. Parameters such as the DIII-D shot number are obtained by means of LabView logging on to an intermediate computer and examining a file which is in fact written by the neutral beam IBM PC which is also connected to the AppleTalk network. The IBM PC records the current DIII-D shot number from the MODCOMP computers.

\section{OPERATION}

One concern was how well this quasi-realtime system would perform while doing other duties, such as running word processing and spreadsheet applications. The system in fact performs extremely well, and is able to acquire and display the data while allowing the neutral beam coordinator to perform their other duties of report writing and availability logging (Fig. 4). The acquisition and display tasks do momentarily slow down the word processor and spread sheet functions during the acquisition and display update. This is probably more desirable than the acquisition being subject to interference, and is only experienced for a brief period after the tokamak shot. The effect is to some degree fortuitous as it is not under the direct control of the programmer. 


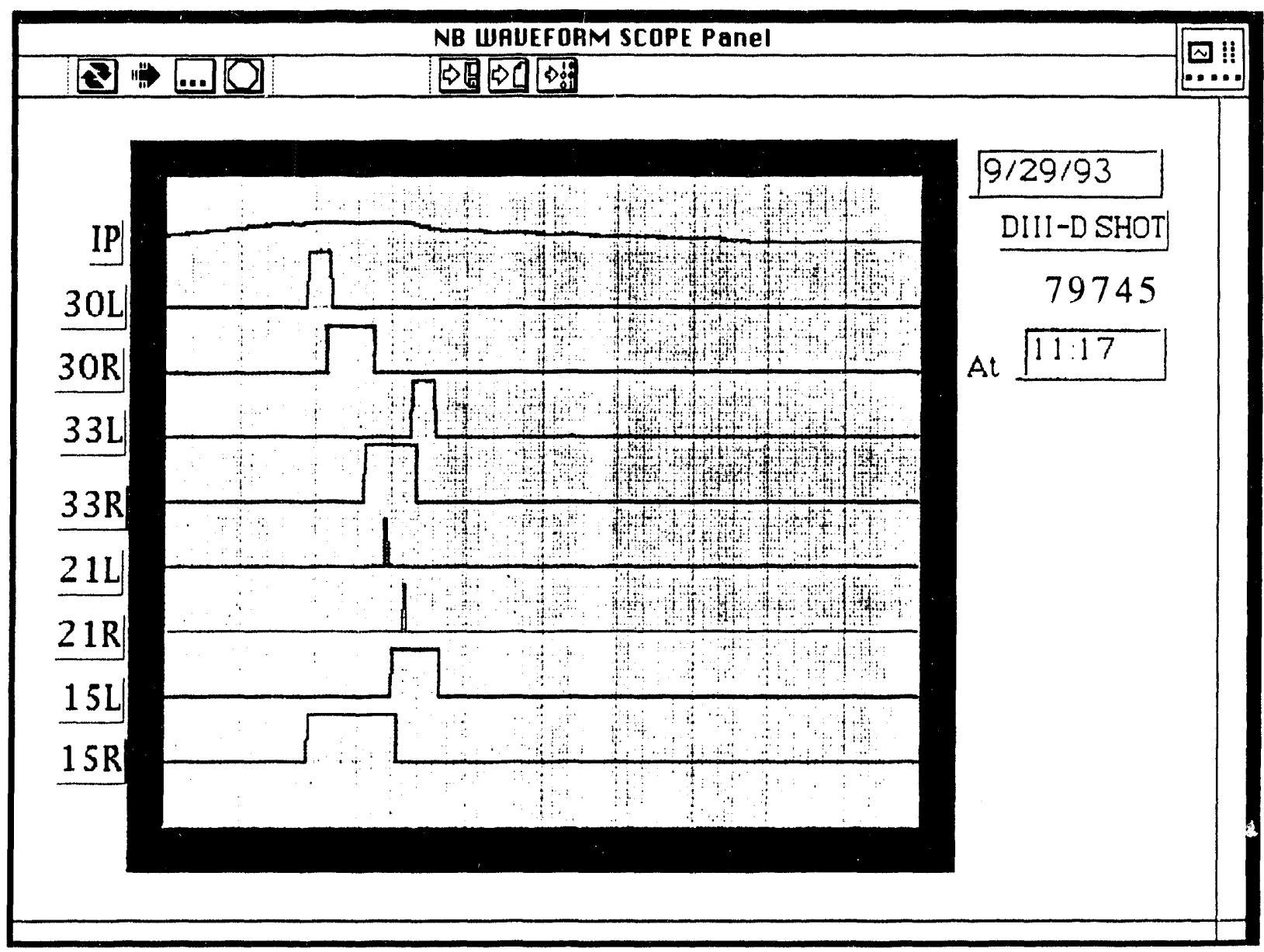

FIg 4. The neutral beam data display is shown. In practice, there is also report writing in Word and statistical logging in Excel on the same screen.

\section{SUMMARY}

The display described here has proven to be very useful to the neutral beam coordinator, and has been relatively inexpensive to implement when compared to the cost of an oscilloscope. Often the costs of setup and programming are underestimated or overlooked with any new system. In this case, they were minimal due to the ease of learning, implementation, and the flexibility of the software used. The initial work for this display has just recently been completed and it is expected that in the future new functions will be added, especially in the area of automatically computing beam availability (requested versus injected beam).

\section{ACKNOWLEDGMENT}

The author gratefully acknowledges the work of Paul Thurgood in developing the neutral beam IBM PC display which showed the value of a centralized display and is the workhorse in terms of writing all the neutral beam MODCOMP data to the VAX cluster each shot.

\section{REFERENCES}

[1] P. A. Henline, "Use of open systems for control, analysis, and data acquisition of the DIII-D tokamak," this symposium.

[2] Paul Thurgood, "PC-link historical data base system MODCOMP/IBM at link for neutral particle bearn operation," in Proc. 13th IEEE Symp. on Fusion Engineering, 1989, p. 1467.

[3] Prodivet Literature. Austin, TX: National Instruments, July 1993. 

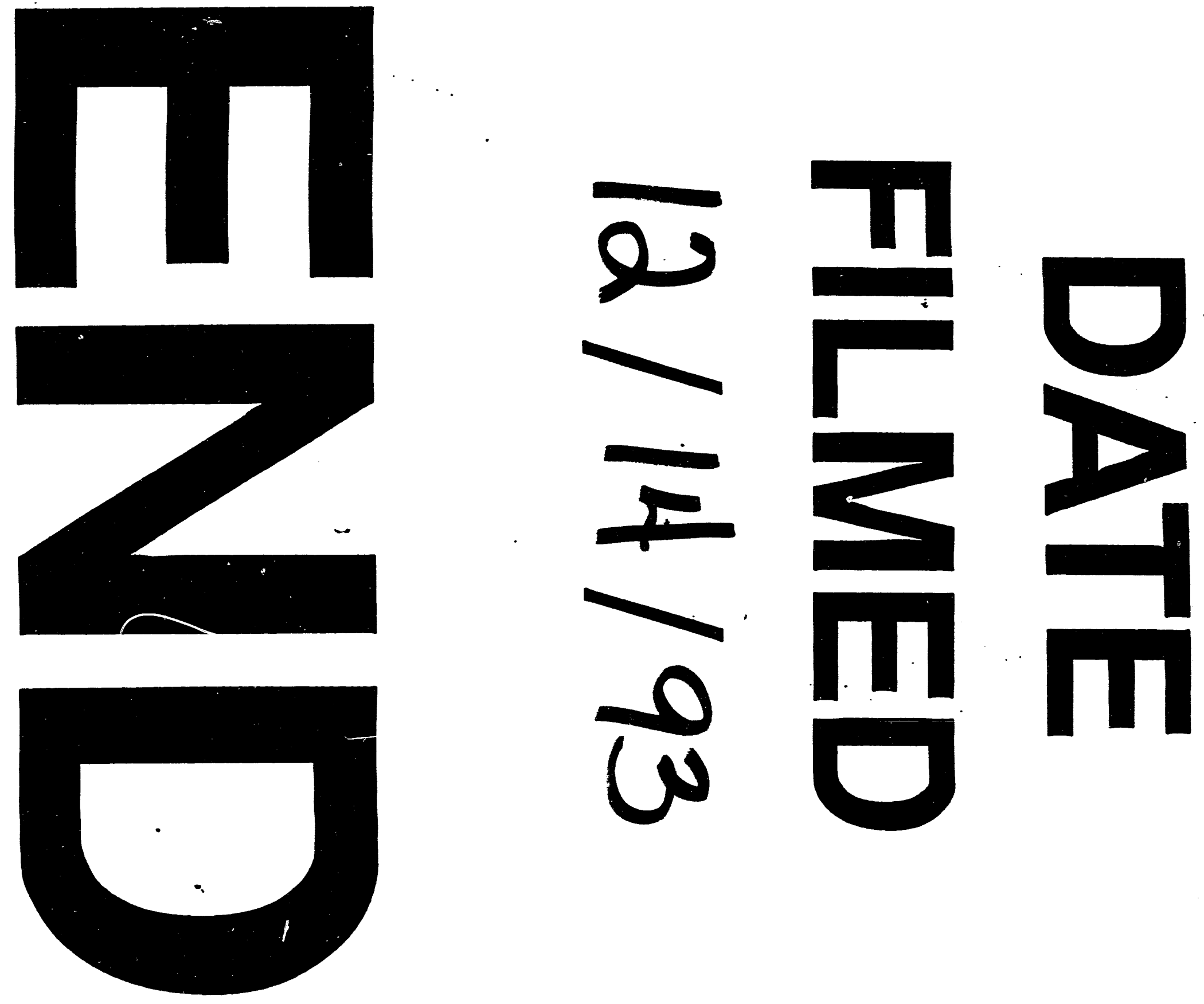


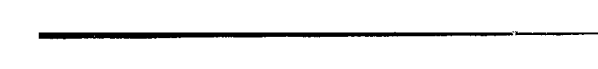

